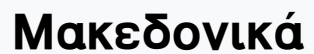

Tóp. 4, Ap. 1 (1960)

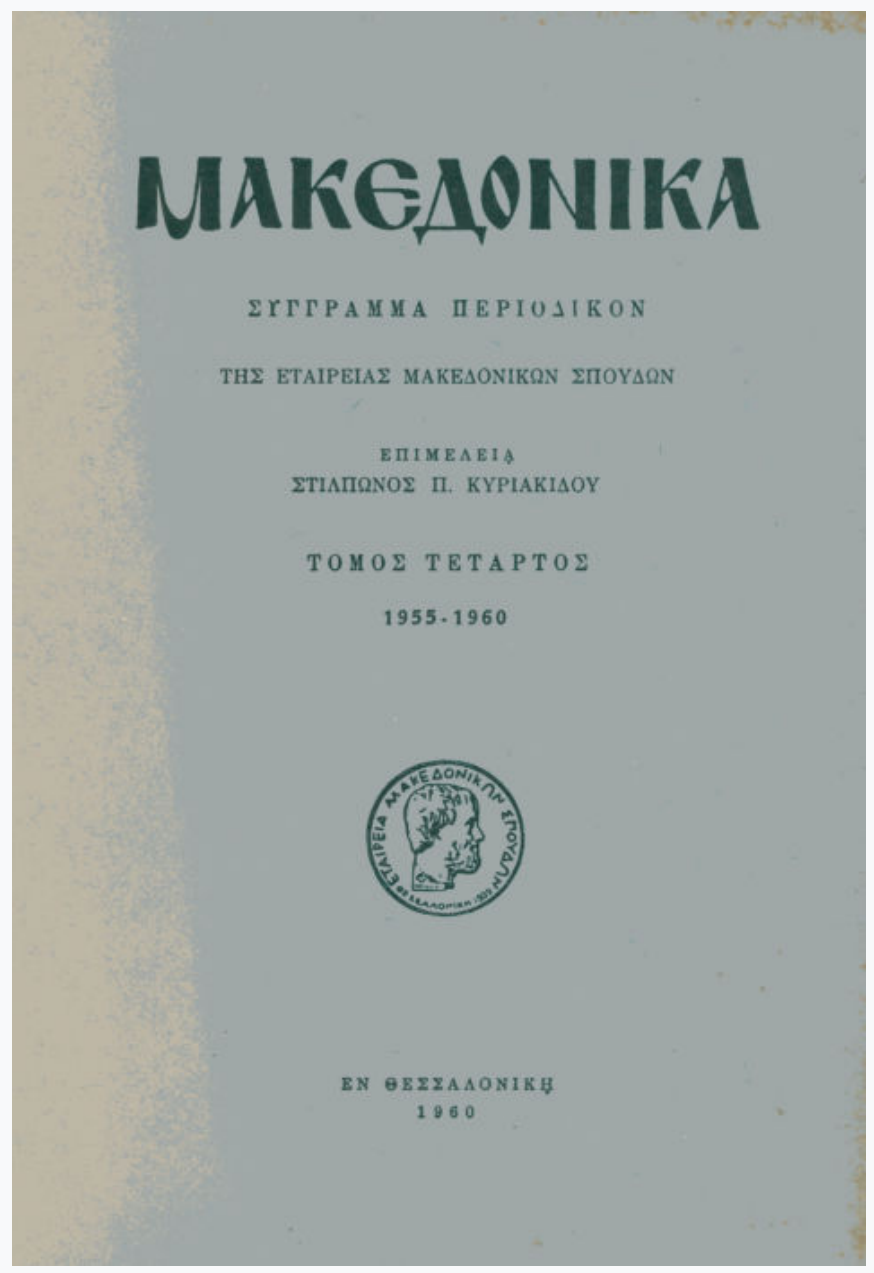

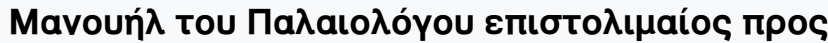

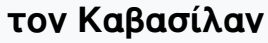

Raymond-J. Loenertz

doi: 10.12681/makedonika.663

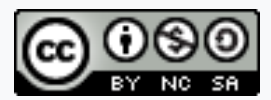

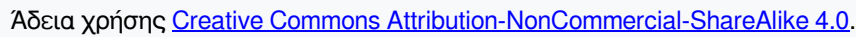

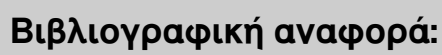

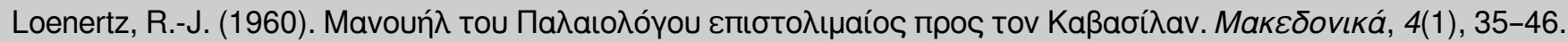
https://doi.org/10.12681/makedonika.663 


\section{MANUEL PALÉOLOGUE, ÉPITRE A CABASILAS}

$L_{i}$ opuscule que nous publions est intitulé dans les manuscrits Discours en forme de lettre à Cabasilas. Cetitre, qui semble remonter à $l^{\prime}$ auteur, pourrait faire penser que le caractère épistolaire y est de pure convention, d'autant plus que Manuel Paléologue l' a exclu du recueil de ses lettres proprement dites. Toutefois la longueur exceptionnelle de $1^{\prime}$ épître explique assez ce traitement, tandis que, pour le fond, et en dépit de certaine apparence didactique, $c^{\prime}$ est bien une lettre très personnelle, et bel échantillon $d^{\prime}$ épistolographie byzantine. Seulement, pour saisir ce caractère personnel il faut être averti des circonstances où se trouvait l'auteur, afin de pouvoir reconnaître les données concrètes qui se cachent sous les énoncés généraux. On peut alors se rendre compte que $1^{\circ}$ épître à $\mathrm{Ca}$. basilas est l'oeuvre d' un homme que la mauvaise fortune poursuit sans $l^{\prime}$ abattre, d'un homme $d^{\prime}$ action qui est aussi un homme de lettres et qui, momentanément condamné à un repos forcé, trouve un dérivatif à ses peines en les racontant à un ami, lettré comme lui, et en $s^{\prime}$ efforçant de le faire en ce beau langage qu' il admire chez ses auteurs favoris.

L' épître à Cabasilas date de $1^{\prime}$ été 1387 . Manuel Paléologue vient de se réfugier dans l' île de Lesbos, où il est arrivé en avril mai, après avoir quitté 'Thessalonique, qu' il avait défendue quatre années contre les assiégeants turcs. ${ }^{1}$ Après son départ la ville capitula. Lui - même avait été contraint au départ à la suite d' une émeute des bourgeois, qui ne voulaient plus subir les rigueurs du siège

1 La date exacte et la durée du siège dans le cod. Marcian. gr. 408,

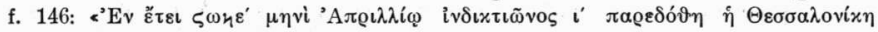

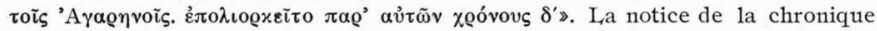
brève thessalonicienne que j'ai publiée dans Les recueils de lettres de Démétrius Cydonès, Vatican, 1947, p. 117 n. 2, se rapporte à un siège de Thessalonique par les Tures en avril $1372,10 \mathrm{e}$ indiction, 15 ans avant la prise de la ville. Je publierai cette chronique en entier dans les appendices au t. I de la Correspondance de Démétrius Cydonès, qui est sous presse. 
ni, comme ils disaient, la «tyrannie» de Manuel, qui les obligeait à résister. Chassé par ses sujets, le prince aurait pû se retirer à Con-

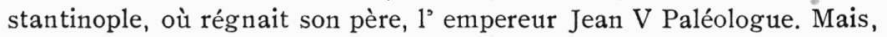
pour comble de malheur, un désaccord sérieux, dont nous ignorons la genèse et devinons à peine les motifs, opposait depuis quelque temps le père et le fils. ${ }^{1} \mathrm{D}^{\prime}$ ailleurs, le vieil empereur était contraint de ménager les Turcs, avec lesquels il maintenait des relations pacifiques à l' époque même où son fils les combattait en Macédoine. Ce $n$ ' est pas sans peine que Manuel et sa suite avaient trouvé un refuge à Lesbos, dont le seigneur, François II Gattilusio, son cousin germain, devait compter aussi avec la susceptibilité de $1^{\circ}$ émir ottoman, Mourad Ier, et ne pas lui fournir prétexte à des hostilités. Et puis il était Génois, et avait partie liée avec ceux qui soutenaient les droits d' Andronic IV, frère ainé de Manuel, sur le trône de Byzance. Andronic était mort en $\mathrm{r} 385$, mais son fils Jean VII revendiquait sa succession, et quelques années plus tard, vraisemblablement en $\mathrm{r} 39^{\circ}$, il devait épouser Eugénie, fille de François. ${ }^{2}$ Notre épître, et plusieurs lettres de Démétrius Cydonès, prouvent que le fugitif fut reçu dans l' île. Mais le dynaste génois $\mathrm{n}^{3}$ admit pas son cousin dans les murs de sa capitale fortifiée, et $l^{\prime}$ on peut croire $\mathrm{qu}^{\prime}$ il le pria de prolonger son séjour le moins possible. De fait, Manuel campa en plein air et

- On trouve une allusion très discrète à ce conflit au chapitre 9 de 1' épitre à Cabasilas; voir aussi les lettres suivantes de Démétrius Cydonès: G. C a m m e 11 i, Démétrius Cydonès. Correspondance, Paris, 1930, nº 30 (à Manuel Paléologue), lin. $86-89 ; n^{\circ} 31$ (à Rhadènos, non à Chrysobergès), lin. 92 - 95. R. J. L o e ner tz, Les recueils, p. 103 - 104 (à Rhadènos), lin. 60 - 69. C a m m e $11 \mathrm{i}$, Op. cit., p. $154 \mathrm{n}^{0} 119$ (à la dernière ligne du sommaire biffer «Andronic ou* et supprimer le?).

${ }^{2}$ Le 26. V. 1390 le sénat de Venise, à la demande des ambassadeurs du comte de Vertus (Jean - Galéas Visconti, seigneur de Milan) autorise 1' impératrice de Constantinople à s'embarquer, à ses propres frais, sur un bâteau vénitien à destination de la Romanie. Le même jour, revenant sur sa décision, il remet la réponse à plus tard, afin d'entendre d'abord les ambassadeurs de 1' impératrice, qui sont à Venise; Venezia, Archivio di Stato, Senato, Secreta E, f. 54v - 55. V. aussi Analele Academiei Române, S. II, t. 36 (1913 - 1914) $1104 \mathrm{n}^{0} \mathrm{x}$ viii (1390. VII. 10). En avril 1390, quand Jean VII

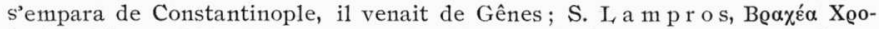
vıxó, Athènes, 1932 - 1933, n ${ }^{0} 15$ (p. 32) 29-31. La présence d' un Manuel Cabasilas à Gênes en janvier 1390 est-elle en rapport avec celle de Jean VII ? Genova Archivio notarile, Pandette Richeriane, B, fasc. 69 c. 3, Quaderno di Teramo Maiolo; cité, K. Hopf, Geschichte Griechenlands etc., Allgemeine Encyklopädie der Wissenschaften und Künste, t. 86, Leipzig, 1868, p. 54 n. 5. 
ne tarda pas à se rendre dans une autre île, déserte-probablement Ténédos-d' où il entra en pourparlers avec l' émir turc. De Ténédos, quand Mourad lui eut promi bon accueil, il passa à Brousse comme le raconte Chalcocondyle, dont le récit est confirmé quant à l' essentiel par les lettres de Démétrius Cydonès. ${ }^{1}$ Telles furent les circonstances où Manuel composa 1' épître à Cabasilas.

Ce dernier n'est pas désigné par son prénom. On nous dit seulement qu'il était de Thessalonique et vivait à Constantinople. Une lettre de Cydonès, écrite après la lecture de l'épître, nous apprend en plus que le destinataire était grand ami de Cydonès. ${ }^{2}$ C' est assez pour rendre probable que notre Cabasilas n'est autre que Nicolas Chamaëtos Cabasilas, le mystique. Il est malheureusement impossible de transformer en certitude cette probabilité.

Nous publions 1' épître d'après le manuscrit 304 I de la Bibliothèque Nationale de Paris, qui a toute l'autorité d'un autographe, puisqu'il a été entre les mains de l'auteur et que celui - ci a corrigé personnellement un des ouvrages qui s'y trouvent, le Di a log u e $\mathrm{sur}$ le mariage, qui occupe les fol. 89- I04 du manuscrit et nous donnons en note les très rares variantes du cod. Barb. gr. 2 I9 (=B), f. $24 \cdot 29^{\mathrm{v}}$.

- Ces lettres se trouvent au fascicule 19 du ms. autographe Vat. gr. 101 , f. $129-136^{\mathrm{v}}$, dans l'ordre où elles furent expédiées ce qui en facilite grandement $1^{\prime}$ intelligence.

${ }^{2} \mathrm{C}$ a m m e $11 \mathrm{i}, \mathrm{n}^{0} 21$. 


\section{MANOYHA TOY ПАМАIOАOГOY

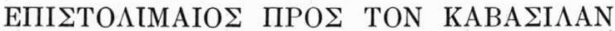

Lesbi, 1387 , aestate

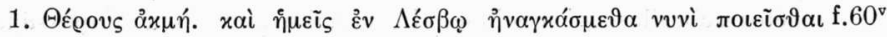

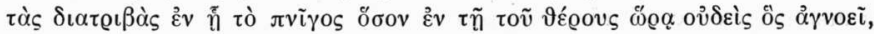

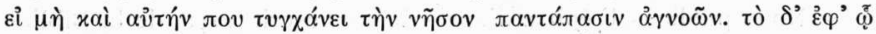

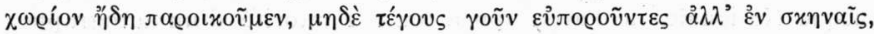

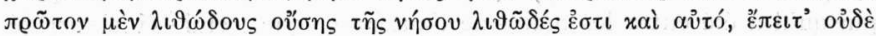

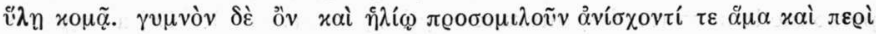

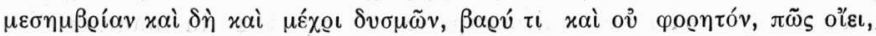

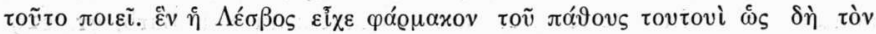

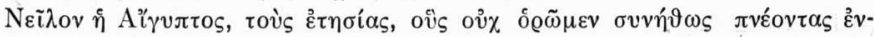

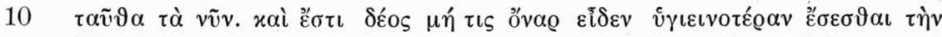

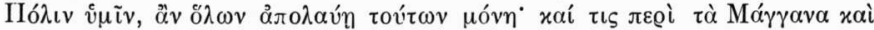

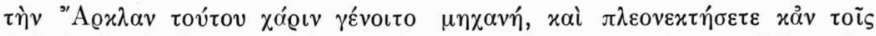

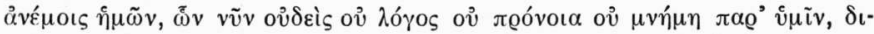

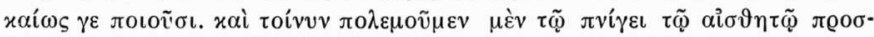

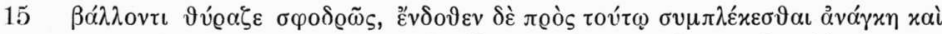

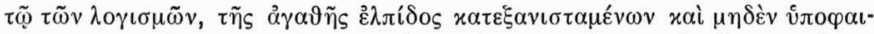

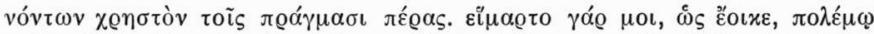

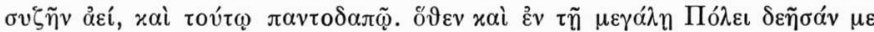

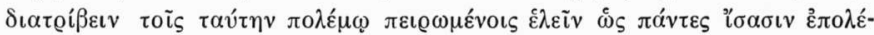

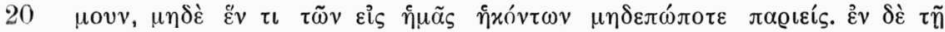

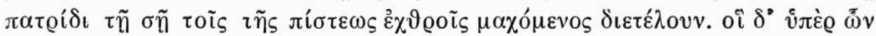

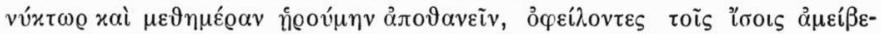
$\sigma \vartheta \alpha \iota$ ทै

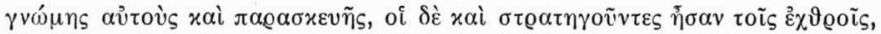

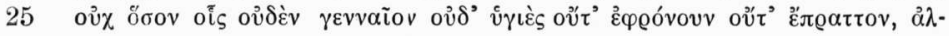

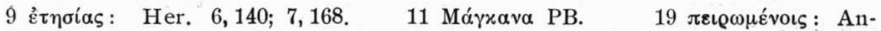

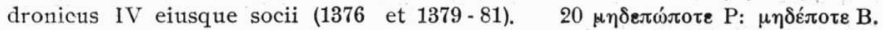

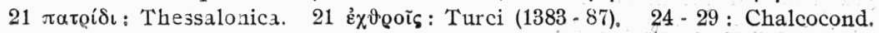




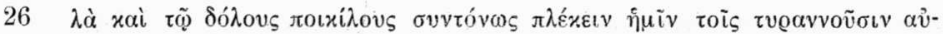

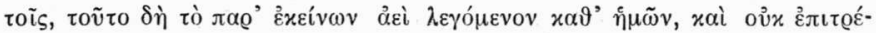

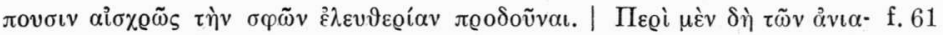

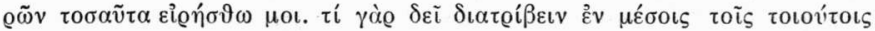

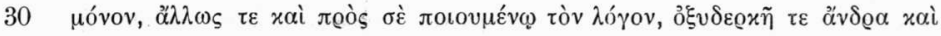

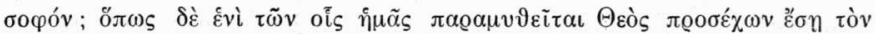

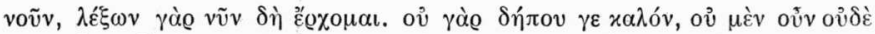

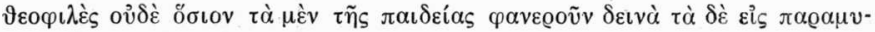

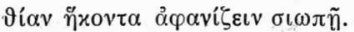

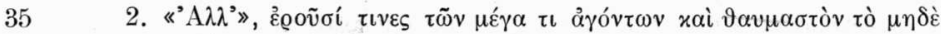

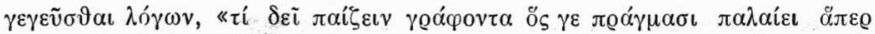

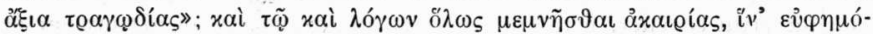

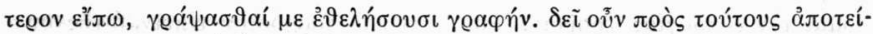

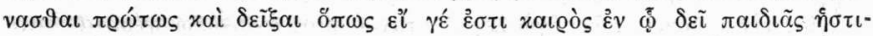

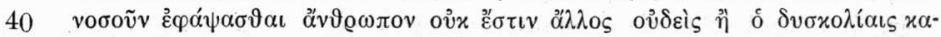

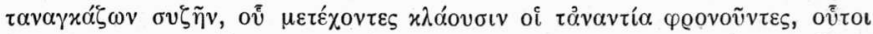

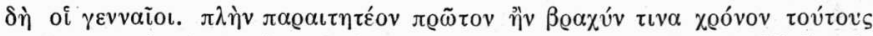

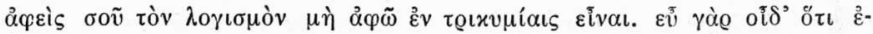

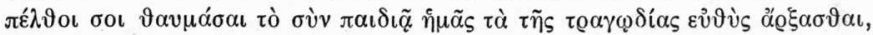

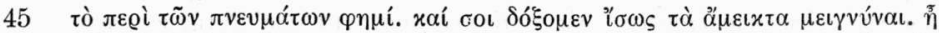

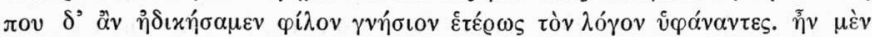

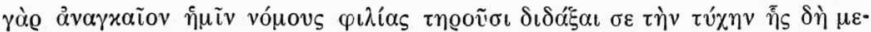

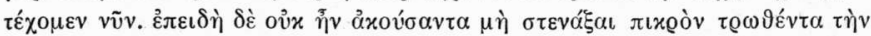

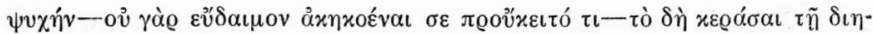

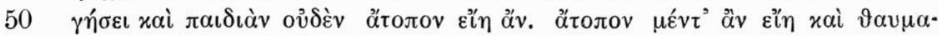

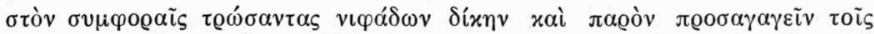

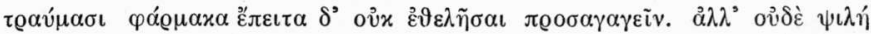

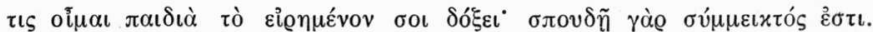

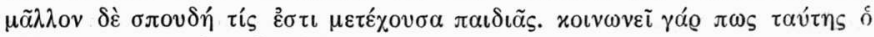

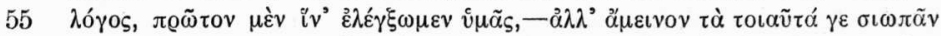
一

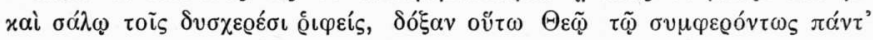

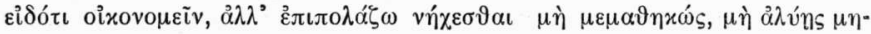

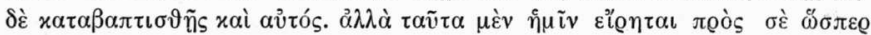

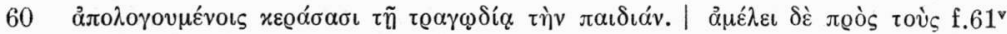

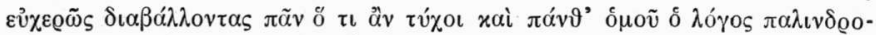

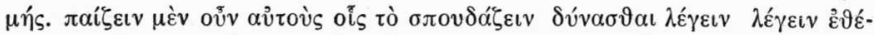

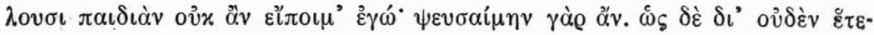

I, p. $33 \cdot 34$ (Darkó). 


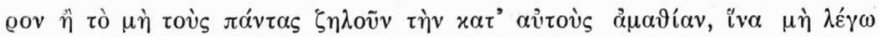

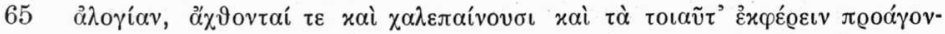

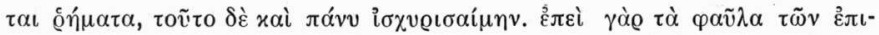

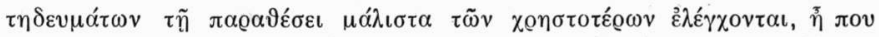

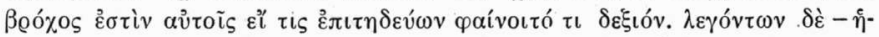

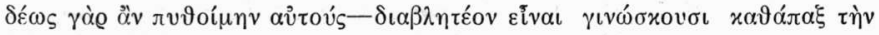

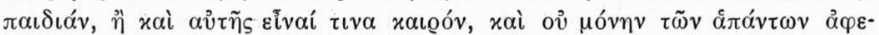

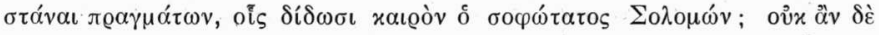

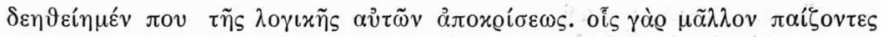

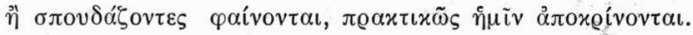

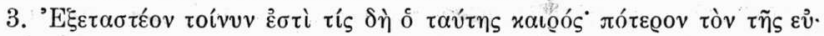

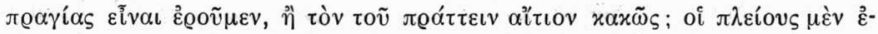

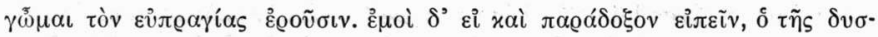

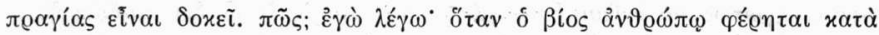

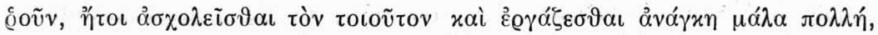

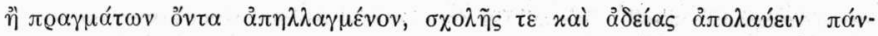

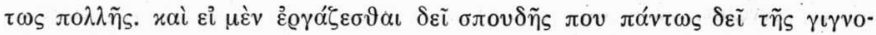

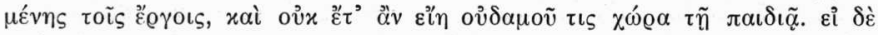

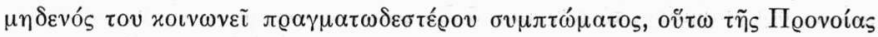

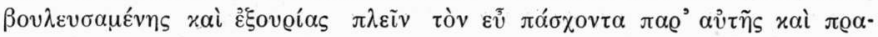

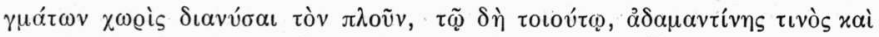

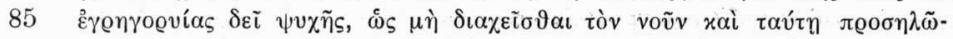

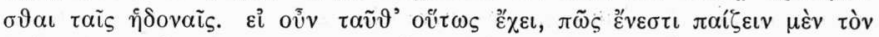

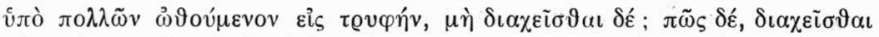

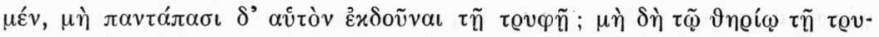

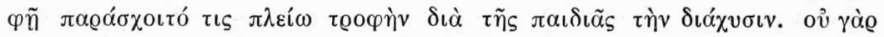

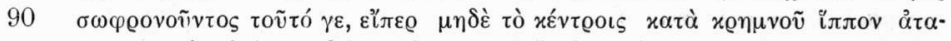

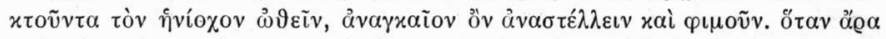

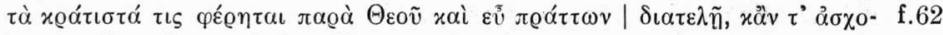

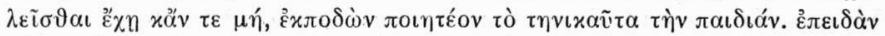

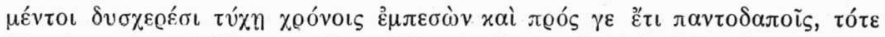

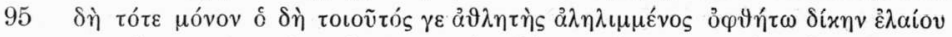

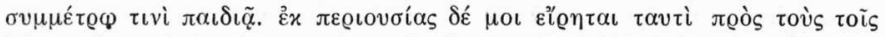
$\lambda$ hó

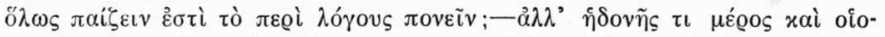

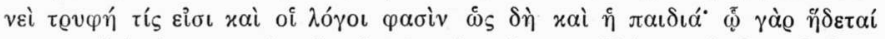

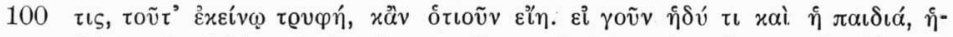

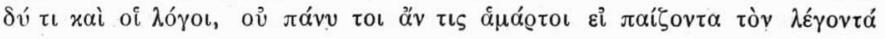

71 Eccl. III 1 - 8. 


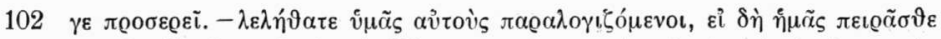

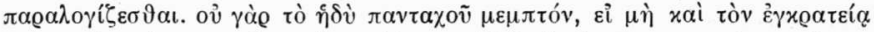

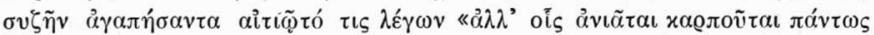

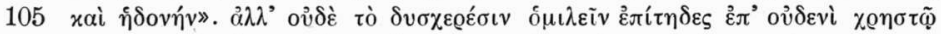

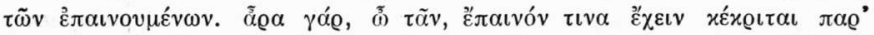

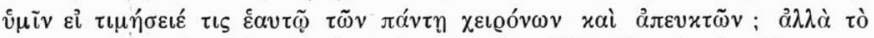

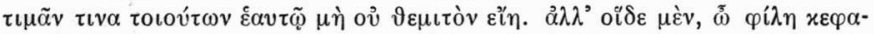

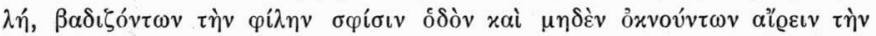

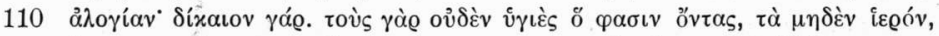

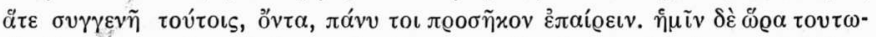

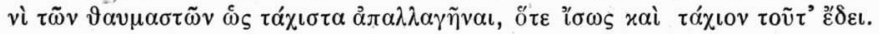

4. 'Н

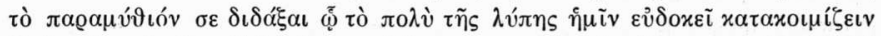

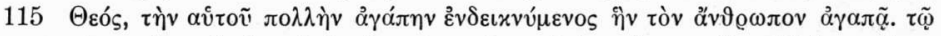

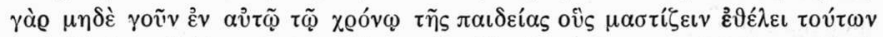

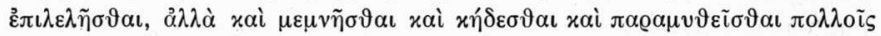

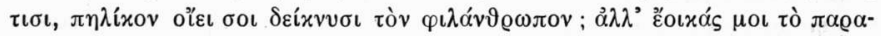

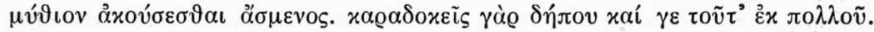

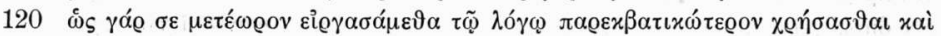

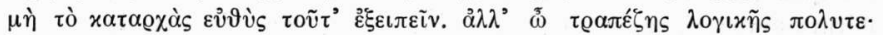

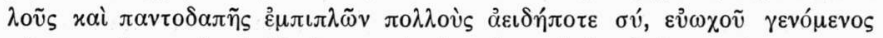

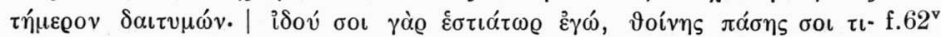

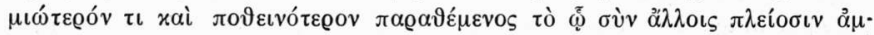

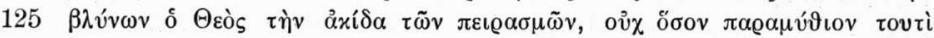

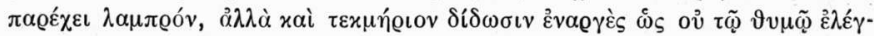

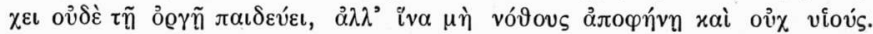

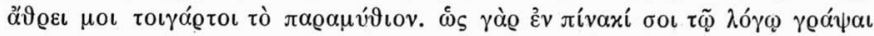

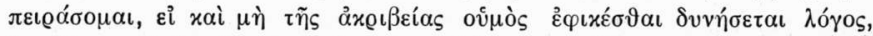

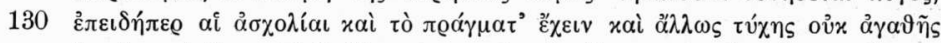

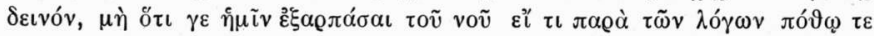

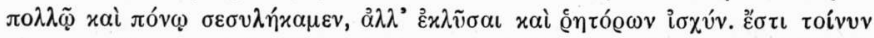

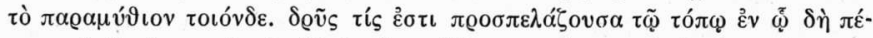

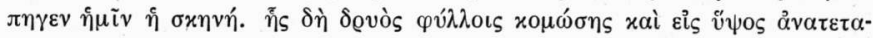

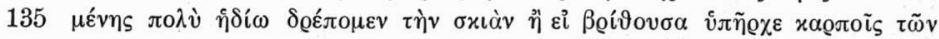

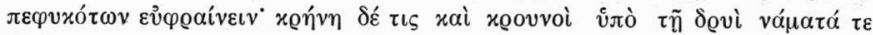

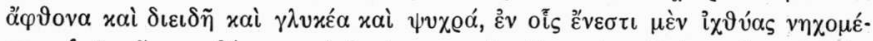
vov

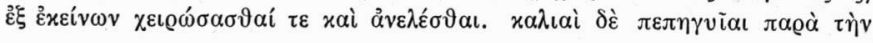

126 - 127 Psalm. VI, 1; XXXVII, 1. 


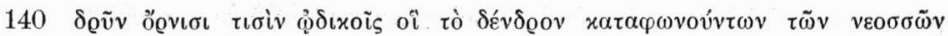

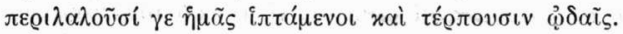

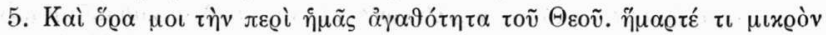

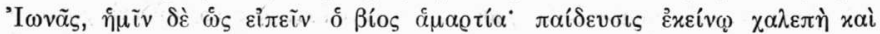

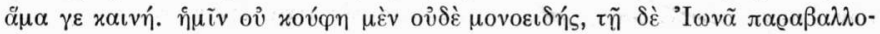

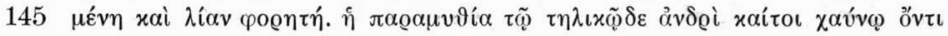

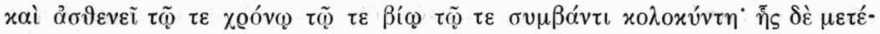

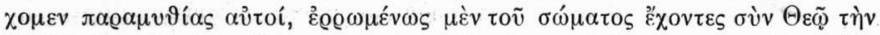

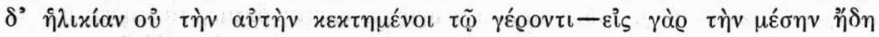

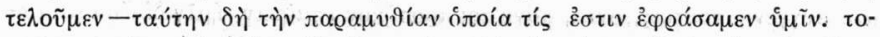

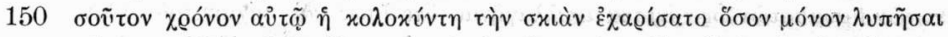
$\tau \tilde{\varphi} \mu \alpha \varrho \alpha v \vartheta \tilde{\eta} v \alpha$.

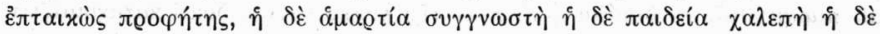

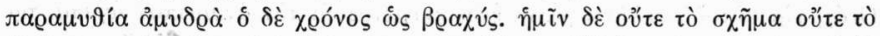

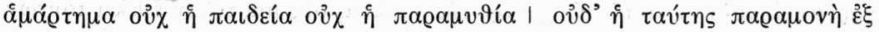

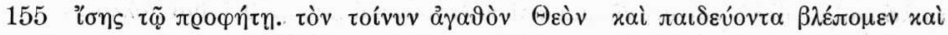

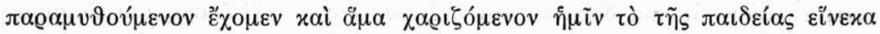

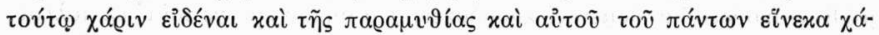

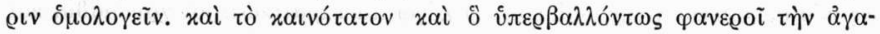

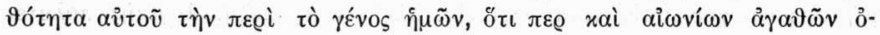

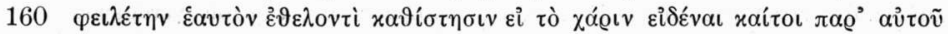

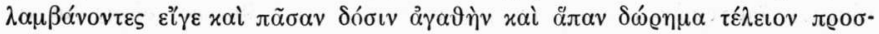

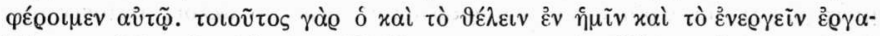

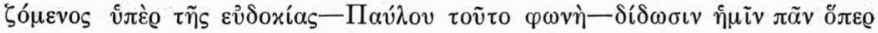

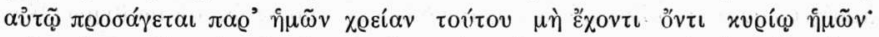

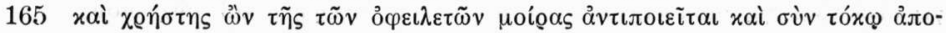

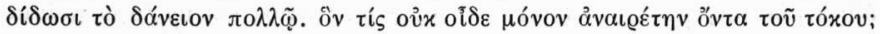

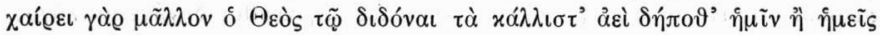

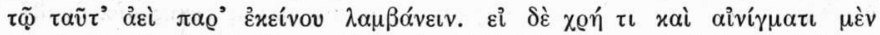

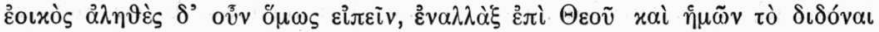

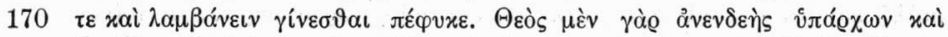

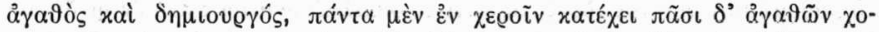

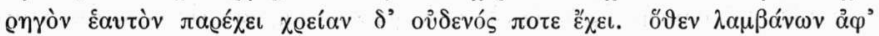

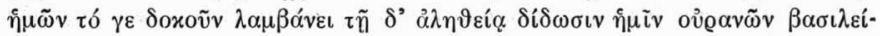

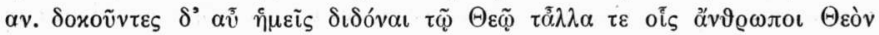

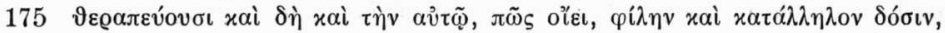

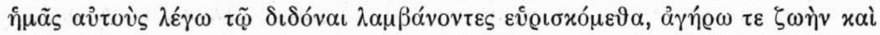

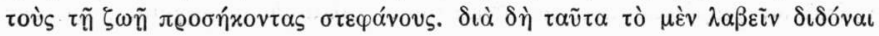

161 - 162 Jac. I 17. 163 Phil. II 13. 


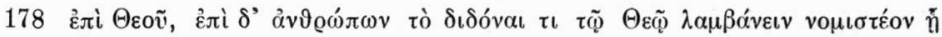

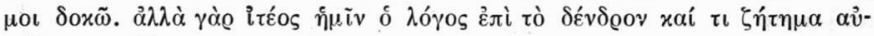

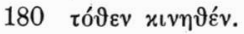

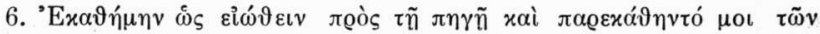

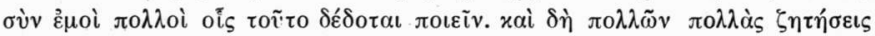

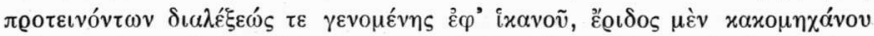

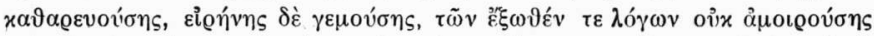

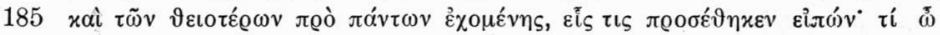

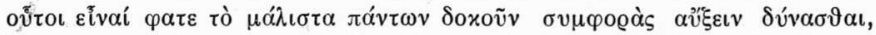

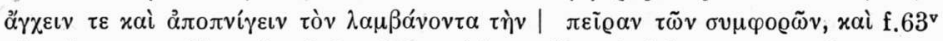

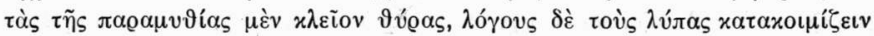

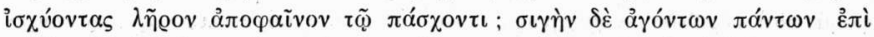

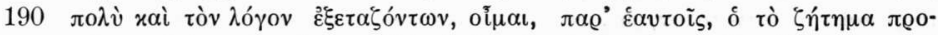

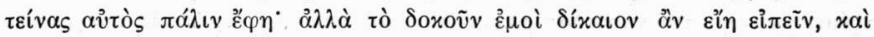

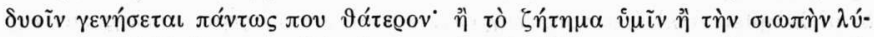

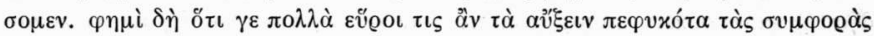

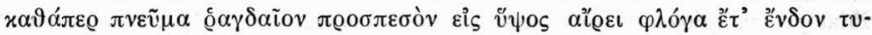

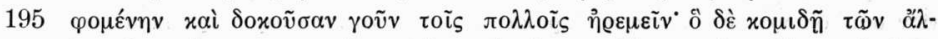

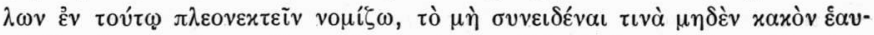

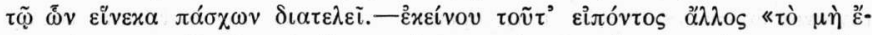

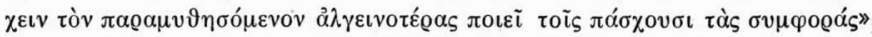

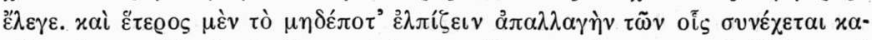

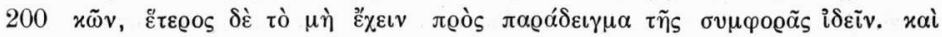

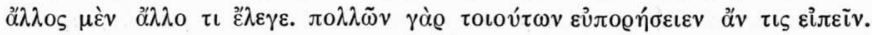

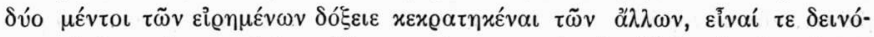

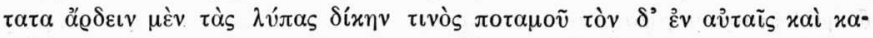

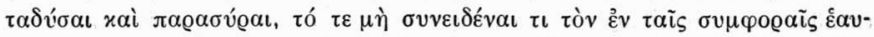

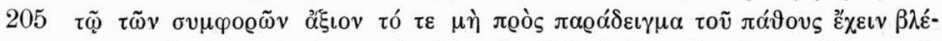

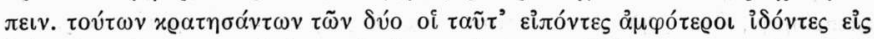

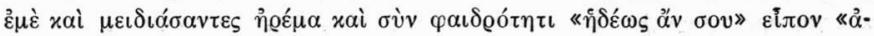

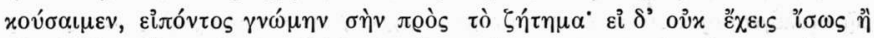

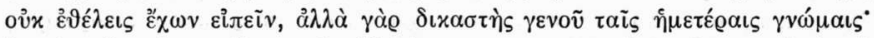

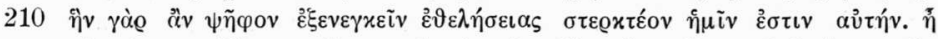

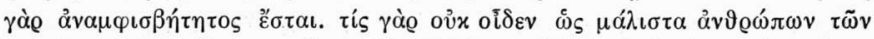

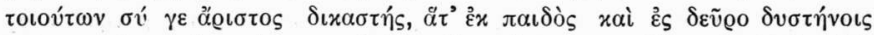

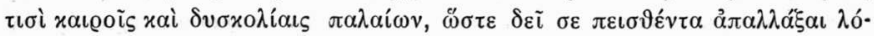

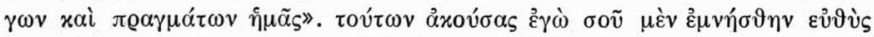

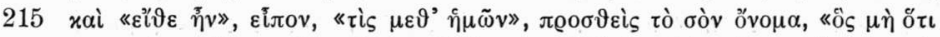

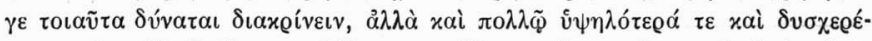

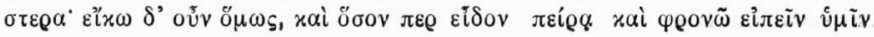




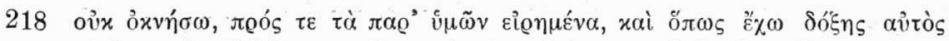

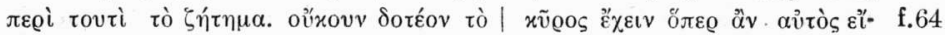

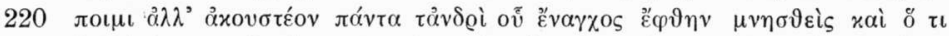

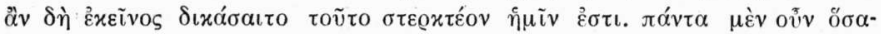

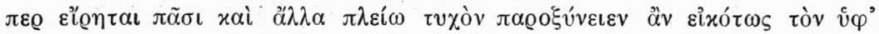

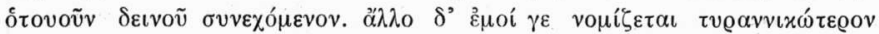

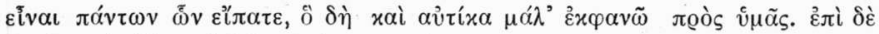

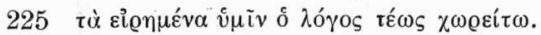

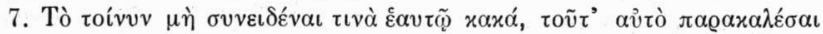

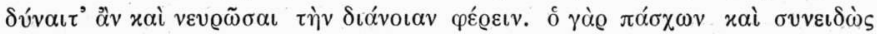

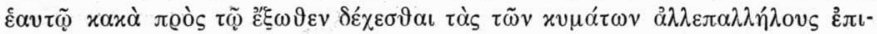

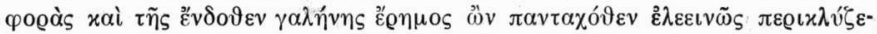

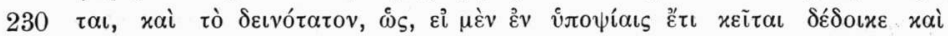

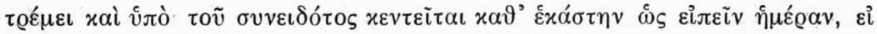

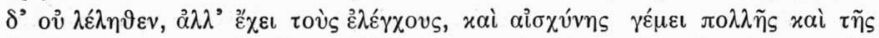

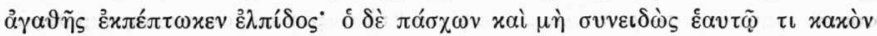

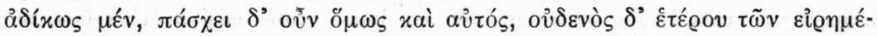

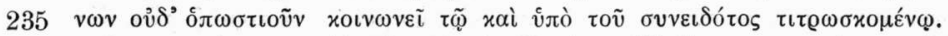

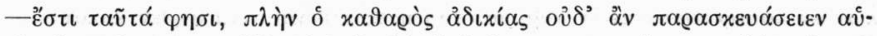

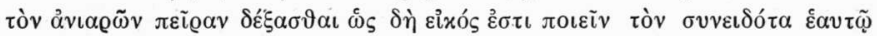

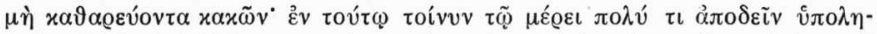

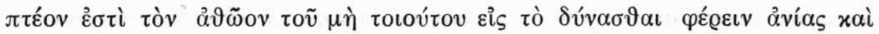

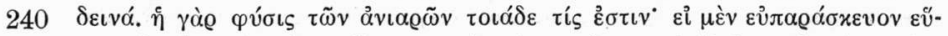

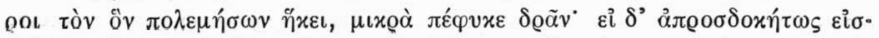

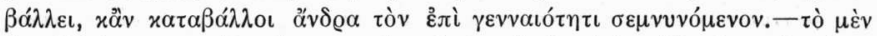

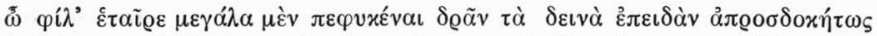

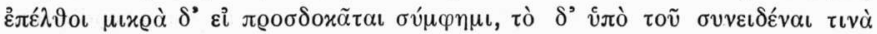

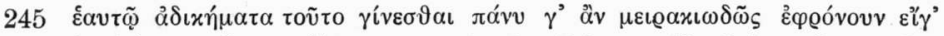

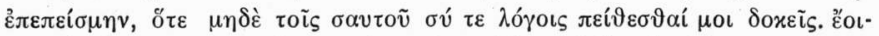

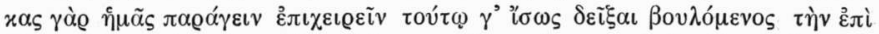

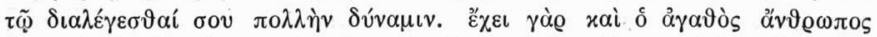

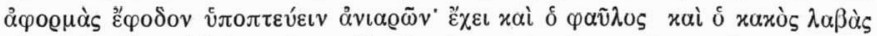

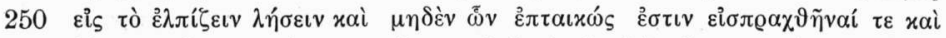

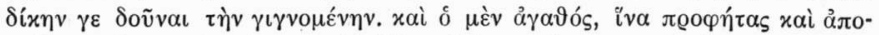

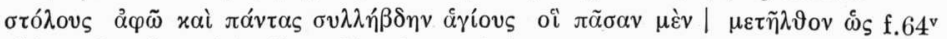

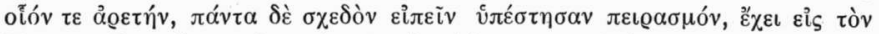

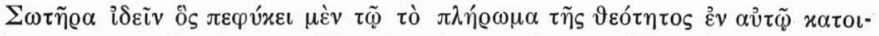

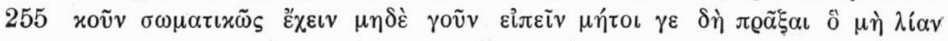

$254-255$ Col. II 9 


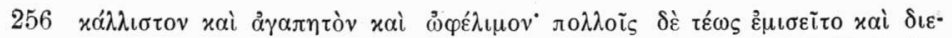

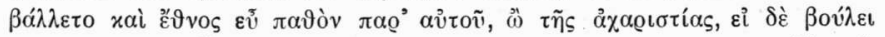

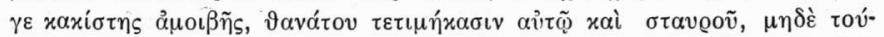

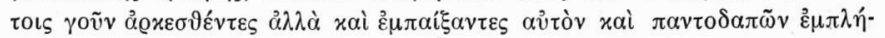

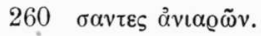

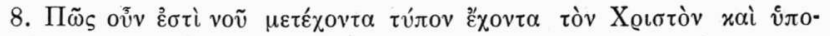

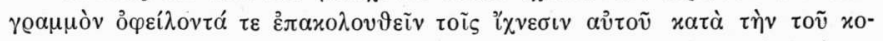

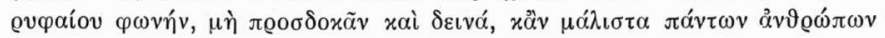

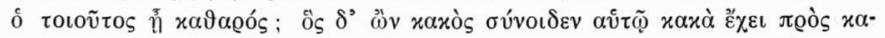

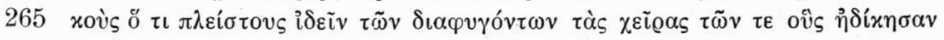

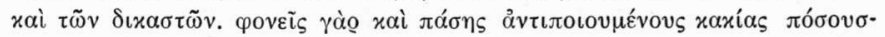

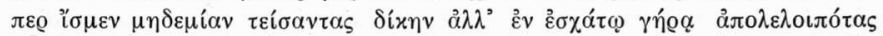

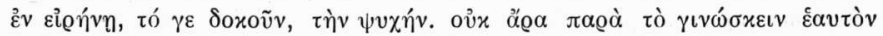

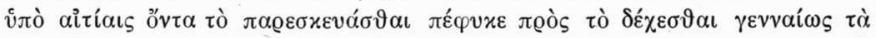

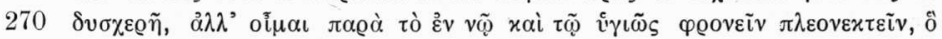

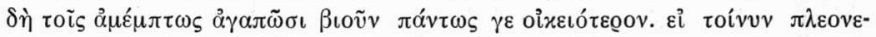

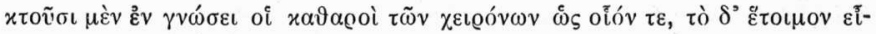

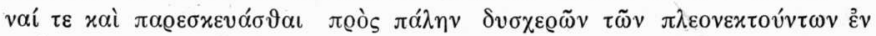

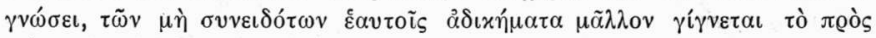

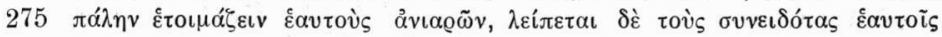

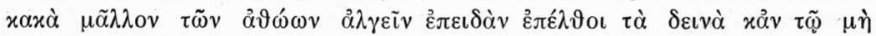

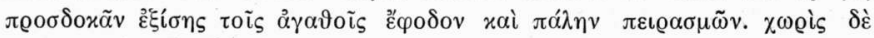

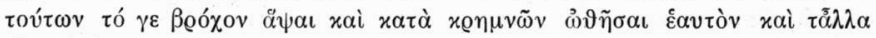

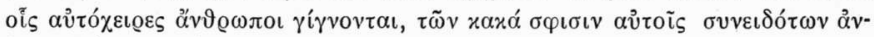

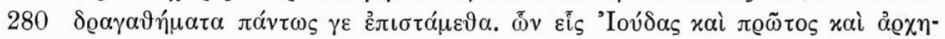

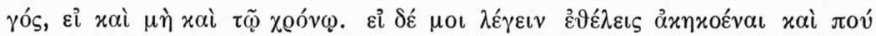

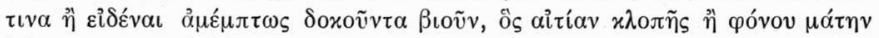

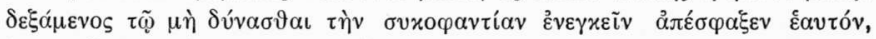

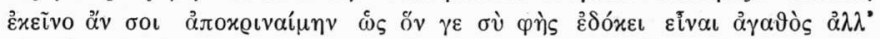

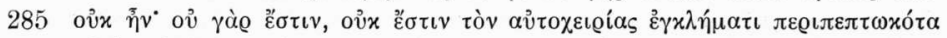

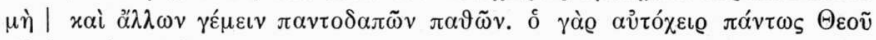

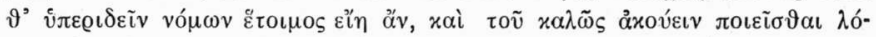

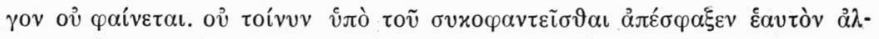

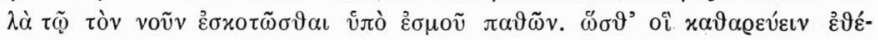

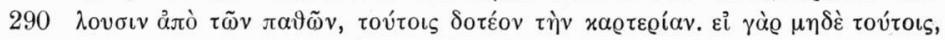

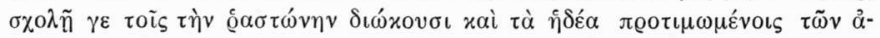

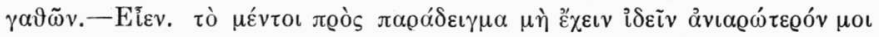

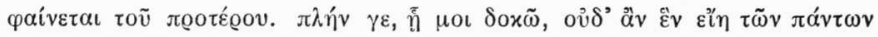

2621 Pet. II 21. 269 aitiass: aitias B $278 \gamma \varepsilon: \tau \varepsilon$ B 


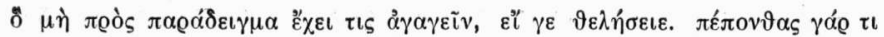

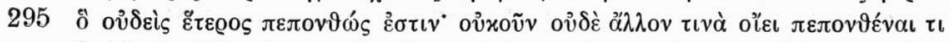

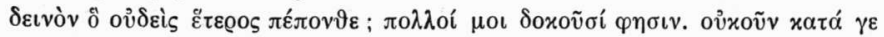

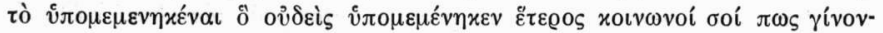

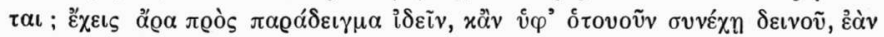

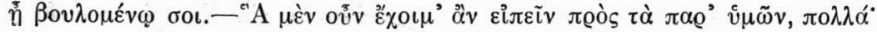

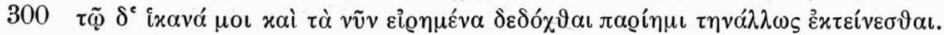

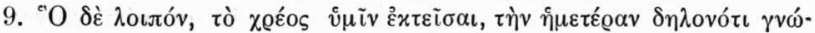

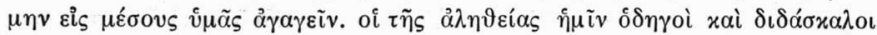

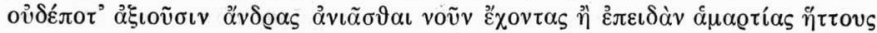

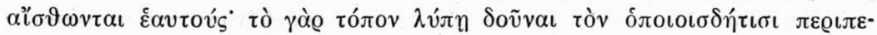

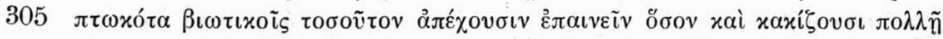

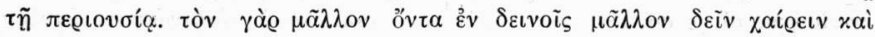

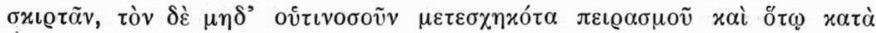

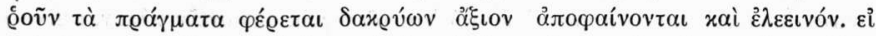

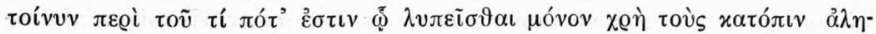

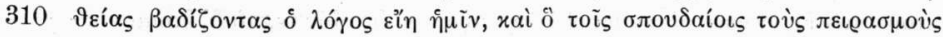

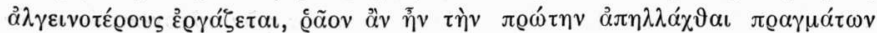

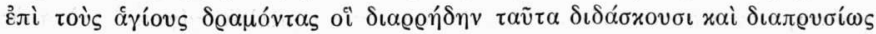

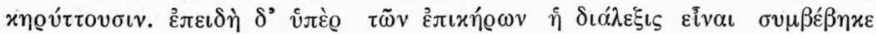

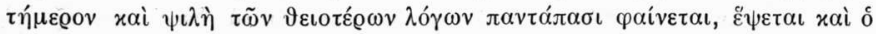

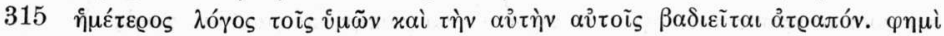

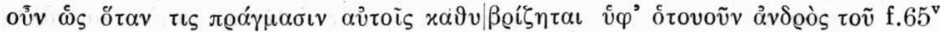

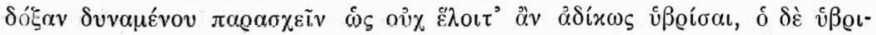

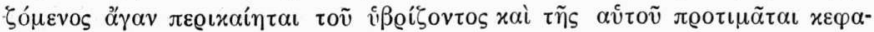

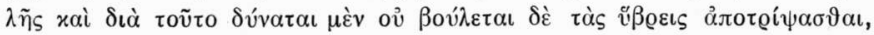

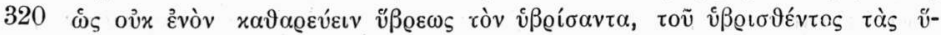

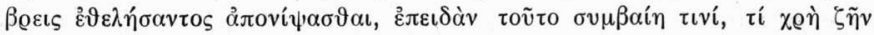

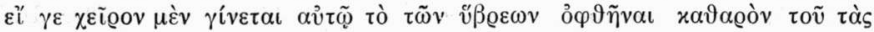

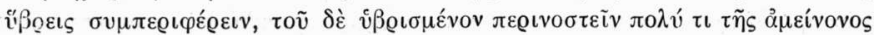

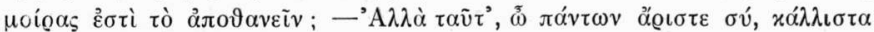

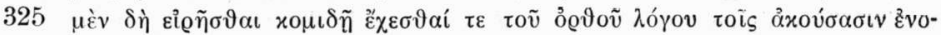

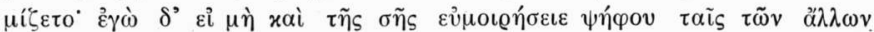

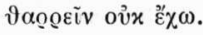

RAYMOND - J. LOENERTZ

316 ảvo̊òs: Joannes Palaeologus Aug., Emmanuelis pater; Chalcocond. I 42, 18 - 21 (Darkó). 